\title{
Propagation of Uncertainty in Dynamical Systems
}

\author{
H.T. Banks ${ }^{1}$ and Shuhua $\mathrm{Hu}^{2}$ \\ Center for Research in Scientific Computation \\ Center for Quantitative Sciences in Biomedicine \\ North Carolina State University \\ Raleigh, NC 27695-8212 USA \\ E-mail: ${ }^{1}$ htbanks@ncsu.edu ${ }^{2}$ shu3@ncsu.edu
}

\begin{abstract}
We compare several approaches to uncertainty propagation that have been used in the literature to formulate the uncertainty in a dynamical system governed by ordinary differential equations. Specifically we focus on the evolution of probability density functions of the associated stochastic processes, and discuss their applications in different fields.
\end{abstract}

\section{Introduction}

Uncertainty is ubiquitous in physics, chemistry, bioscience and especially in the social science. It is often classified into two types. One is epistemic (or reducible) uncertainty, which can possibly be reduced by improved measurements. The other is aleatory (or irreducible) uncertainty, which is the result of intrinsic variability/stochasticity of the system. Uncertainty propagation through a dynamic system has enjoyed considerable research interests during the past decade due to the wide applications of mathematical models in studying the dynamical behavior of the system.

In this paper, we consider two types of differential equations in the presence of uncertainty. One is stochastic differential equations (SDEs), a classification reserved for differential equations driven by white noise. The other is random differential equations (RDEs), a classification used for differential equations driven by colored noise. Specifically, we focus on the evolutions of the probability density functions of stochastic processes resulting from SDEs and RDEs, and their applications in different fields.
Unless otherwise indicated, a capital letter is used to denote a random variable, a bold capital letter is for a random vector, and their corresponding small letters are for their realizations throughout.

\section{Stochastic Differential Equations}

White noise is widely used in practice to account for the randomness in the inputs of system governed by differential equations, especially in the case where one has little knowledge about the precise nature of the noise. In this section, we consider stochastic differential equations driven by Gaussian white noise. Note that stochastic differential equations can be interpreted in either Itô or Stratonovich sense, and the difference between their solutions disappears if the drift term is corrected based on the so-called Wong-Zakai theorem (e.g., see [11, Section 3.4]). Hence, in this section we focus our discussions on the Itô stochastic differential equations.

Consider the following Itô stochastic differential equations

$$
\begin{aligned}
& d \mathbf{X}(t)=\mathbf{g}(t, \mathbf{X}(t)) d t+\boldsymbol{\sigma}(t, \mathbf{X}(t)) d \mathbf{W}(t), \\
& \mathbf{X}(0)=\mathbf{X}_{0},
\end{aligned}
$$

where $\mathbf{X}=\left(X_{1}, X_{2}, \ldots, X_{n}\right)^{T}, \quad \mathbf{g}=$ $\left(g_{1}, g_{2}, \ldots, g_{n}\right)^{T}$ is a nonrandom $n$-dimensional function of $t$ and $\mathbf{x}, \boldsymbol{\sigma}$ is a nonrandom $n \times l$ matrix function of $t$ and $\mathbf{x}, \mathbf{W}(t)$ is an $l$ dimensional standard Wiener process independent of the random initial vector $\mathbf{X}_{0}$. In addition, we assume that $\mathbf{g}$ and $\boldsymbol{\sigma}$ satisfy conditions that guarantee the existence and uniqueness of 
solutions to initial value problem (2.1).

Itô stochastic differential equations are often found useful in financial mathematics as it only takes into account the information about the past (for example, in modeling the stock price, the only information one has is about past events). Itô stochastic differential equations have also been used in describing mechanisms of climate variability. For example, it was used in [20] to describe the interactions between the atmosphere temperature and the ocean's surface temperature.

The evolution of the probability density function of the solution to (2.1) is described in the following theorem.

Theorem 2.1. Assume that $\mathbf{X}_{0}$ has probability density function $p_{0}$, and $\mathbf{X}(t)$ satisfies (2.1). Then the probability density function $p$ of $\mathbf{X}(t)$ satisfies

$$
\begin{aligned}
& \frac{\partial}{\partial t} p(t, \mathbf{x})+\sum_{k=1}^{n} \frac{\partial}{\partial x_{k}}\left(g_{k}(t, \mathbf{x}) p(t, \mathbf{x})\right) \\
& =\frac{1}{2} \sum_{k, j=1}^{n} \frac{\partial^{2}}{\partial x_{k} \partial x_{j}}\left[\left(\boldsymbol{\sigma}(t, \mathbf{x}) \boldsymbol{\sigma}^{T}(t, \mathbf{x})\right)_{k j} p(t, \mathbf{x})\right]
\end{aligned}
$$

with initial condition $p(0, \mathbf{x})=p_{0}(\mathbf{x})$. Here $\left(\boldsymbol{\sigma}(t, \mathbf{x}) \boldsymbol{\sigma}^{T}(t, \mathbf{x})\right)_{k j}$ denotes the $(k, j)$ th element of matrix $\boldsymbol{\sigma}(t, \mathbf{x}) \boldsymbol{\sigma}^{T}(t, \mathbf{x})$.

Equation (2.2) is often referred to as FokkerPlanck equation or forward Kolmogorov equation, and is important to the fields of chemistry and thermodynamics. Fokker-Planck equations have also been used in the literature (e.g., $[3,8]$ ) to describe the population density in a sizestructured population where the growth process is a diffusion process satisfying the Itô stochastic differential equation. There are several approaches that can be used to derive the Fokker-Planck equation. One approach is to employ expansion methods such as those in [14, Section 8.1] employed by Moyal in 1949. Another method is based on the principle of preservation of probability density functions (e.g., see $[10]$ ).

\section{Random Differential Equations}

Colored noise has been observed in many settings such as astronomy, acoustic equipment and electronic devices. Hence, random differential equations have gained considerable research interest in the past decade, especially the efforts on computational methods (interested readers can refer to [23] for discussions on widely used methods such as Monte Carlo methods, stochastic Galerkin methods and probabilistic (or stochastic) collocation methods). In practice, one often decomposes and truncates the colored noise by a finite linear combination of uncorrelated random variables (e.g., using a Karhunen-Loève expansion) for uncertainty quantification. Hence, in this section we focus our discussions on those random differential equations with random inputs characterized by finite dimensional random vectors.

\subsection{Differential Equations with Ran- dom Initial Conditions}

In this section, we consider the evolution of probability density function of the solution to a system of ordinary differential equation with random initial conditions

$$
\dot{\mathbf{x}}=\mathbf{g}(t, \mathbf{x}), \quad \mathbf{x}(0)=\mathbf{X}_{0},
$$

where $\mathbf{x}=\left(x_{1}, x_{2}, \ldots, x_{n}\right)^{T}$, and $\mathbf{g}=$ $\left(g_{1}, g_{2}, \ldots, g_{n}\right)^{T}$ is an $n$-dimensional nonrandom function of $t$ and $\mathbf{x}$. Here $\mathbf{X}_{0}$ is an $n$ dimensional random vector. Equation (3.1) is referred to as a crypto-deterministic system (e.g., see $[11,14,18]$ ), and has been proven useful in wide applications such as classical statistical mechanics, statistical thermodynamics, kinetic theory and biosciences. Depending on the applications, the uncertainty in initial conditions could be classified as either epistemic uncertainty (e.g., the uncertainty is due to the measurement error) or aleatory uncertainty (e.g., individuals have different initial size).

Theorem 3.1. [18, Theorem 6.2.2] Assume that (3.1) has a mean square solution $\mathbf{x}\left(t ; \mathbf{X}_{0}\right)$. 
Then the probability density function of the solution $\mathbf{x}\left(t ; \mathbf{X}_{0}\right)$ satisfies

$$
\frac{\partial}{\partial t} p(t, \mathbf{x})+\sum_{k=1}^{n} \frac{\partial}{\partial x_{k}}\left(g_{k}(t, \mathbf{x}) p(t, \mathbf{x})\right)=0
$$

with initial condition $p(0, \mathbf{x})=p_{0}(\mathbf{x})$, where $p_{0}$ is the probability density function of $\mathbf{X}_{0}$.

The above result is often referred to as $L i$ ouville's "theorem", and the resulting equation (3.2) is often called Liouville's equation. Liouville's "theorem" is a key result in statistical mechanics and statistical thermodynamics. It is worth noting that Liouville's equation with $n=3$ is the well-know equation of continuity in continuum mechanics. There are several methods that can be used to derive this equation. One is from a probabilistic point of view using the concept of characteristic functions (e.g., see [18, p147]) following the derivation of Kozin [12] in 1961. Another method is based on the principle of preservation of probability density function [16, pages 363-364]. In addition, we see that (3.1) is a special case of Itô stochastic differential equation (2.1) with $\boldsymbol{\sigma} \equiv 0$. Hence, if we assume that $\mathbf{g}$ satisfies the conditions for existence and uniqueness of solutions of (2.1), then we can use the same arguments as those for deriving the Fokker-Planck equation to derive Liouville's equation.

\subsubsection{Application of Liouville's Equa- tion in Population Dynamics}

We observe that the Liouville equation (3.2) with $g_{1} \equiv 1$ is a special case of a model given by Oster and Takahashi in 1974 [15] ${ }^{1}$

$$
\begin{aligned}
& \frac{\partial}{\partial t} u(t, \mathbf{x})+\frac{\partial}{\partial x_{1}} u(t, \mathbf{x}) \\
& +\sum_{j=2}^{n} \frac{\partial}{\partial x_{j}}\left(g_{j}(t, \mathbf{x}) u(t, \mathbf{x})\right)+d(t, \mathbf{x}) u(t, \mathbf{x})=0
\end{aligned}
$$

without mortality $(d=0)$. This model is used to describe the population density

\footnotetext{
${ }^{1}$ The connection between the Liouville equation and (3.3) was recognized by Oster and Takahashi in the case of zero mortality and constant growth rates $g_{i}, i=2, \ldots, n$.
}

$u(t, \mathbf{x})$ in a spatially homogeneous population where individuals are characterized by $\mathbf{x}=$ $\left(x_{1}, x_{2}, \cdots, x_{n}\right)^{T}$ with $x_{1}$ denoting the chronological age and $x_{2}, \cdots, x_{n}$ representing some physiological variables such as mass, volume, chemical composition, and any other quantities having an influence on individual's growth and mortality rates, and $g(t, \mathbf{x})$ and $d(t, \mathbf{x})$ are the growth and mortality rates of individuals with chronological age $x_{1}$ and physiological variables $x_{2}, \ldots, x_{n}$ at time $t$, respectively.

Equation (3.3) includes a number of wellknown structured population models as special cases. Specifically, the model with only chronological age involved, that is,

$$
\frac{\partial}{\partial t} u(t, x)+\frac{\partial}{\partial x} u(t, x)+d(t, x) u(t, x)=0
$$

is the age-structured population model given by Mckendrick [13] in 1926 and Von Foerster [21] in 1959. Equation (3.3) with $n=2$,

$$
\begin{aligned}
& \frac{\partial}{\partial t} u(t, \mathbf{x})+\frac{\partial}{\partial x_{1}} u(t, \mathbf{x})+\frac{\partial}{\partial x_{2}}\left(g_{2}(t, \mathbf{x}) u(t, \mathbf{x})\right) \\
& +d(t, \mathbf{x}) u(t, \mathbf{x})=0
\end{aligned}
$$

is the age-size structured population model developed by Sinko and Streifer [17] in 1967 (with $x_{2}$ being some physiological variable), and it is also the model given by Bell and Anderson [9] in 1967 for cell populations (with $x_{2}$ denoting the volume of the cell). Equation (3.4) without the second term, that is,

$$
\begin{gathered}
\frac{\partial}{\partial t} u(t, x)+\frac{\partial}{\partial x}(g(t, x) u(t, x)) \\
+d(t, x) u(t, x)=0,
\end{gathered}
$$

is often referred to as the Sinko-Streifer model or classical size-structured population model. Here $x$ is the structure variable, which may represent weight, length, volume, chronological age (equation (3.5) becomes an age-structured population model in this case), caloric content, maturity, etc, depending on the applications.

Based on the above discussions, we see that all the linear structured population models presented in this section can be associated with some stochastic process, which is obtained due to the variability in the initial size of individuals in the population. 


\subsection{Differential Equations with Ran- dom Model Parameters and Ran- dom Initial Conditions}

In this section, we consider the system of random differential equations

$$
\dot{\mathbf{x}}=\mathbf{g}(t, \mathbf{x} ; \mathbf{Z}), \quad \mathbf{x}(0)=\mathbf{X}_{0},
$$

where $\mathbf{x}=\left(x_{1}, x_{2}, \ldots, x_{n}\right)^{T}, \quad \mathbf{g}=$ $\left(g_{1}, g_{2}, \ldots, g_{n}\right)^{T}$ is an $n$-dimensional nonrandom function of $t$ and $\mathbf{x}, \mathbf{Z}$ is an $m$-dimensional random vector with values in $\mathbb{Z}$, and $\mathbf{X}_{0}$ is an $n$-dimensional random vector.

Equation (3.6) has wide applications in physics and bioscience to account for heterogeneity of the material or the variation between individuals (and the uncertainty is aleatory in this case). For example, a system of ordinary differential equations are often used to model the HIV dynamics for any specific individual, but the model parameters vary across the patients as the clinical data shows a great deal of variability among HIV patients (e.g., see $[2,7]$ ). Equation (3.6) can also be used to describe the uncertainty arising from the parameter estimation error, and in this case the uncertainty is epistemic.

\subsubsection{Evolution of Joint Proba- bility Density Function of $\left(\mathbf{x}\left(t ; \mathbf{X}_{0}, \mathbf{Z}\right), \mathbf{Z}\right)^{T}$}

In this section, we consider the evolution of probability density functions of $\left(\mathbf{x}\left(t ; \mathbf{X}_{0}, \mathbf{Z}\right), \mathbf{Z}\right)^{T}$. Let $\tilde{\mathbf{x}}=(\mathbf{x}, \mathbf{z})^{T}$. Then (3.6) can be rewritten as

$$
\dot{\tilde{\mathbf{x}}}=\tilde{\mathbf{g}}(t, \tilde{\mathbf{x}}), \quad \tilde{\mathbf{x}}(0)=\left(\mathbf{X}_{0}, \mathbf{Z}\right)^{T} .
$$

Here $\tilde{\mathbf{g}}(t, \tilde{\mathbf{x}})=\left[\begin{array}{c}\mathbf{g}(t, \mathbf{x} ; \mathbf{z}) \\ \mathbf{0}_{m}\end{array}\right]$, where $\mathbf{0}_{m}$ is an $m$-dimensional column vector with all the elements being zeros. Hence, Theorem 3.1 can be applied to (3.7) to obtain the following result on the evolution of joint probability density functions of the solution to (3.7).

Theorem 3.2. [19, Theorem 1] Assume that (3.6) has a mean square solution $\mathbf{x}\left(t ; \mathbf{X}_{0}, \mathbf{Z}\right)$.
Then the joint probability density function $\tilde{\varphi}_{\mathbf{X}, \mathbf{Z}}(t, \mathbf{x}, \mathbf{z})$ of $\mathbf{x}\left(t ; \mathbf{X}_{0}, \mathbf{Z}\right)$ and $\mathbf{Z}$ satisfies

$$
\begin{aligned}
& \frac{\partial}{\partial t} \tilde{\varphi} \mathbf{X}, \mathbf{Z}(t, \mathbf{x}, \mathbf{z}) \\
& \quad+\sum_{k=1}^{n} \frac{\partial}{\partial x_{k}}\left(g_{k}(t, \mathbf{x} ; \mathbf{z}) \tilde{\varphi}_{X, \mathbf{Z}}(t, \mathbf{x}, \mathbf{z})\right)=0,
\end{aligned}
$$

with initial condition $\tilde{\varphi}_{\mathbf{X}, \mathbf{Z}}(0, \mathbf{x}, \mathbf{z})=$ $\tilde{\varphi}_{\mathbf{X}, \mathbf{Z}}^{0}(\mathbf{x}, \mathbf{z})$, where $\tilde{\varphi}_{\mathbf{X}, \mathbf{Z}}^{0}$ is the joint probability density function of $\mathbf{X}_{0}$ and $\mathbf{Z}$.

Observe that (3.7) is a special case of stochastic differential equations with diffusion coefficient being zeros. Hence, we can also use the same arguments for deriving the Fokker-Planck equation to derive (3.8).

Given the joint probability density function $\tilde{\varphi}(t, \mathbf{x}, \mathbf{z})$, we can obtain the probability density function $p(t, \mathbf{x})$ for $\mathbf{x}\left(t ; \mathbf{X}_{0}, \mathbf{Z}\right)$ given by

$$
p(t, \mathbf{x})=\int_{\mathbb{Z}} \tilde{\varphi}(t, \mathbf{x}, \mathbf{z}) d \mathbf{z} .
$$

\subsubsection{Evolution of Conditional Prob- ability Density Function of $\mathbf{x}\left(t ; \mathbf{X}_{0}, \mathbf{Z}\right)$ Given the Value $\mathbf{z}$ of $\mathbf{Z}$}

In this section, we derive the conditional probability density function of $\mathbf{x}\left(t ; \mathbf{X}_{0}, \mathbf{Z}\right)$ given the value $\mathbf{z}$ of $\mathbf{Z}$. Note that the conditional probability density function $\varphi(t, \mathbf{x} ; \mathbf{z})$ is

$$
\varphi(t, \mathbf{x} ; \mathbf{z})=\frac{\tilde{\varphi}_{\mathbf{X}, \mathbf{Z}}(t, \mathbf{x}, \mathbf{z})}{\tilde{\varphi}_{\mathbf{Z}}(\mathbf{z})}
$$

where $\tilde{\varphi}_{\mathbf{Z}}(\mathbf{z})$ denotes the probability density function of $\mathbf{Z}$. Hence, by (3.8) and (3.10) we find $\varphi(t, \mathbf{x} ; \mathbf{z})$ satisfies

$$
\frac{\partial}{\partial t} \varphi(t, \mathbf{x} ; \mathbf{z})+\sum_{k=1}^{n} \frac{\partial}{\partial x_{k}}\left(g_{k}(t, \mathbf{x} ; \mathbf{z}) \varphi(t, \mathbf{x} ; \mathbf{z})\right)=0,
$$

with initial condition $\varphi(0, \mathbf{x} ; \mathbf{z})=\varphi_{0}(\mathbf{x} ; \mathbf{z})$, where $\varphi_{0}(\mathbf{x} ; \mathbf{z})$ is the probability density function of initial condition $\mathbf{X}_{0}$ given $\mathbf{Z}=\mathbf{z}$.

Observe that for any given value $\mathbf{z}$ of $\mathbf{Z}$ system (3.6) is crypto-deterministic. Hence, by using Liouville's equation (3.2) the probability density function $\varphi(t, \mathbf{x} ; \mathbf{z})$ for the solution 
$\mathbf{x}\left(t ; \mathbf{X}_{0}, \mathbf{z}\right)$ to (3.6) with given value $\mathbf{z}$ of $\mathbf{Z}$ satisfies (3.11). Thus, we can derive evolution of the conditional probability density function $\varphi(t, \mathbf{x} ; \mathbf{z})$ directly from Liouville's equation.

Given the conditional probability density function $\varphi(t, \mathbf{x} ; \mathbf{z})$, the probability density function $p(t, \mathbf{x})$ of $\mathbf{x}\left(t ; \mathbf{X}_{0}, \mathbf{Z}\right)$ is given by

$$
p(t, \mathbf{x})=\int_{\mathbb{Z}} \varphi(t, \mathbf{x} ; \mathbf{z}) \tilde{\varphi}_{\mathbf{Z}}(\mathbf{z}) d \mathbf{z} .
$$

We remark that the scalar case of (3.11) along with some proper boundary and initial conditions along with (3.12) have been used to describe the population density in a size-structured population, where the entire population is partitioned into subpopulations with each subpopulation having its own growth rate $g(t, x ; \mathbf{z})$. This model, referred to as growth rate distributed size-structured (GRDSS) population model, was first formulated in $[1,6]$ in 1986, and has been successfully used to model mosquitofish population in the rice fields, where the data exhibits both bimodality and dispersion in size as time increases (e.g., see [1]). In addition, this model was also used to model the early growth of shrimp populations, which exhibits a great deal of variability in size as time evolves even though the shrimp begin with approximately similar size $[3,4]$. Based on the above discussions, we see that the GRDSS model can be associated with some stochastic process, which is obtained due to the variability in the individual's growth rate and also the variability in the initial size of individuals in the population. This is made precise in $[5,8]$.

\section{Concluding Remarks}

In this note we consider the evolution of probability density functions of the solutions of Itô SDEs and RDEs as well as their applications in different fields. Even though SDEs and RDEs are quite different in nature (one driven by white noise while the other driven by colored noise), it was shown in [8] that there are classes of Itô SDEs with solutions having the same probability density functions at each time $t$ as those for the solutions of a related corresponding RDE. More precisely, it is shown how to map the SDE to a corresponding RDE and conversely. In addition, based on these pointwise equivalence results it was demonstrated in [5] that the solution for the GRDSS model can be used to approximate the solution of Fokker-Planck equation (which is difficult to solve when the drift dominates the diffusion, the case of primary interest in many cases). However, it should be noted that the stochastic processes obtained by these SDEs and their corresponding RDEs are different as their covariance functions are not the same (see [8] for more details).

\section{Acknowledgements}

This research was supported in part by the National Institute of Allergy and Infectious Diseases under Grant Number R01AI071915-09 and in part by the U.S. Air Force Office of Scientific Research under grant number FA955009-1-0226.

\section{References}

[1] H.T. Banks, L.W. Botsford, F. Kappel and C. Wang, Modeling and estimation in size structured population models, LCDS-CCS Report 87-13, Brown University; Proc. 2nd Course on Math. Ecology, (Trieste, December 8-12, 1986) World Press, Singapore, 1988, 521-541.

[2] H.T. Banks, M. Davidian, S. Hu, G.M. Kepler and E.S. Rosenberg, Modeling HIV immune response and validation with clinical data, J. Biological Dynamics, 2 (2008), 357-385.

[3] H.T. Banks, J.L. Davis, S.L. Ernstberger, S. Hu, E. Artimovich, A.K. Dhar and C.L. Browdy, A comparison of probabilistic and stochastic formulations in modeling growth uncertainty and variability, $J$. Biological Dynamics, 3 (2009), 130-148. 
[4] H.T. Banks, J.L. Davis, S.L. Ernstberger, S. Hu, E. Artimovich and A.K. Dhar, Experimental design and estimation of growth rate distributions in sizestructured shrimp populations, Inverse Problems, 25 (2009), 095003(28pp).

[5] H.T. Banks, J.L. Davis and S. Hu, A computational comparison of alternatives to including uncertainty in structured population models, CRSC-TR09-14, June, 2009; in Three Decades of Progress in Systems and Control, X. Hu, et al, (Eds.), Springer, 2010, 19-33.

[6] H.T. Banks and B.G. Fitzpatrick, Estimation of growth rate distributions in size structured population models, Quart. Appl. Math, 49 (1991), 215-235.

[7] H.T. Banks, S.L. Grove, S. Hu and Y. Ma, A hierarchical Bayesian approach for parameter estimation in HIV models, Inverse Problems, 21 (2005) 1803-1822.

[8] H.T. Banks and S. Hu, Nonlinear stochastic markov processes and modeling uncertainty in populations, CRSC-TR1102, NCSU, January, 2011; Math. Biosci. Engr., to appear.

[9] G.I. Bell and E.C. Anderson, Cell growth and division I. a mathematical model with applications to cell volume distributions in mammalian suspension cultures, Biophysical J., 7 (1967), 329-351.

[10] J. Chen and J. Li, A note on the principle of pervation of probability and probability density evolution equation, Probabilistic Engr. Mech., 24 (2009), 51-59.

[11] T.C. Gard, Introduction to Stochastic Differential Equations, Marcel Dekker, New York, 1988.

[12] F. Kozin, On the probability densities of the output of some random systems, Trans. ASME Ser. E J. Appl. Mech., 28 (1961), 161-164.
[13] A.G. McKendrick, Applications of mathematics to medical problems, Proc. Edinburgh Math. Soc., 44 (1926), 98-130.

[14] J.E. Moyal, Stochastic processes and statistical physics, J. Royal Stat. Soc. Series B (Methodological), 11 (1949), 150-210.

[15] G. Oster and Y. Takahashi, Models for age-specific interactions in a periodic environment, Ecological Monographs, 44 (1974), 483-501.

[16] T.L. Saaty, Modern Nonlinear Equations, Dover, Mineola, N.Y., 1981.

[17] J. Sinko and W. Streifer, A new model for age-size structure of a population, Ecology, 48 (1967), 910-918.

[18] T.T. Soong, Random Differential Equations in Science and Engineering, Academic Press, New York and London, 1973.

[19] T.T. Soong and S.N. Chuang, Solutions of a class of random differential equations, SIAM J. Appl. Math., 24 (1973), 449-459.

[20] G.K. Vallis, Mechanisms of climate variability from years to decades, in Stochastic Physics and Climate Modelling, (edited by T. Palmer and P. Williams), Cambridge University Press, Cambridge, 2010, 1-34.

[21] H. Von Foerster, Some remarks on changing populations, in The Kinetics of Cellular Proliferation, F. Stohlman, Jr. (ed.), Grune and Stratton, New York, 1959.

[22] G.H. Weiss, Equation for the age structure of growing populations, Bull. Math. Biophy., 30 (1968), 427-435.

[23] D. Xiu, Numerical Methods for Stocahstic Computations: A Spectral Method Approach, Princeton University Press, Princeton, NJ, 2010. 